Review Article

\title{
Artificial intelligence: future impacts, challenges and recommendations on healthcare services
}

\author{
Deepkumar Patel*, Shruti Ashok Kore
}

Department of Health Policy and Management, New York Medical College, New York, USA

Received: 30 January 2020

Revised: 08 March 2020

Accepted: 09 March 2020

\section{*Correspondence:}

Dr. Deepkumar Patel,

E-mail: pateldeep21@icloud.com

Copyright: (C) the author(s), publisher and licensee Medip Academy. This is an open-access article distributed under the terms of the Creative Commons Attribution Non-Commercial License, which permits unrestricted non-commercial use, distribution, and reproduction in any medium, provided the original work is properly cited.

\begin{abstract}
In this report, we review the market impact of artificial intelligence (AI) in healthcare and future predictions. AI is a rapidly advancing technology in healthcare. It provides rich and relevant information to patients and healthcare providers with on-demand medical and clinical confidence, AI can greatly advance healthcare professional and patient communications. Interest and investment in artificial intelligence continues to grow. At the same time there exists some practical challenges that will determine the course of this market trend. We will discuss the macroeconomic, ethical and legal challenges that pertain to this industry and make recommendations to the healthcare executives.
\end{abstract}

Keywords: Artificial intelligence, Medical ethics, Health economics, Population health

\section{INTRODUCTION}

Artificial intelligence (AI) is defined as "the use of computer systems able to perform tasks that normally require human intelligence, such as visual perception, speech recognition, decision-making, and translation between languages". ${ }^{1}$ Although AI simulates human decision-making, it does not replace human intelligence; rather, it relies on human training! Unlike other types of technology, AI understands natural language. Natural language is written-out text, rather than structured data such as dates, numbers, and facts that computers normally handle. By understanding natural language and generating hypotheses based on evidence and learning as it goes, AI technology can be trained to make more logical decisions. $^{2}$

\section{HEALTHCARE AI MARKET FORECAST}

In healthcare, according to a new report from Tractica, AI is largely being implemented as a tool to more efficiently and accurately review data and detect patterns in the data that can be used to improve analyses uncover inefficiencies, and streamline care, from both a clinical an operational perspective. The market intelligence firm finds that the overarching driver in these AI implementations is to provide better patient outcomes by reducing morbidity and mortality while controlling the costs and administrative bottlenecks to provide healthcare services. $^{3}$

Tractica forecasts that global software revenue from 21 key healthcare AI use cases will grow from \$165 million in 2017 to $\$ 5.6$ billion annually by 2025 . Including the hardware and services sales that will be driven by these software implementations, the firm anticipates that the total revenue opportunity for the healthcare AI market will reach $\$ 19.3$ billion by 2025 . $^{3}$

In addition, the improving global economy is expected to take a step further in the years ahead and catalyzed the growth of AI in healthcare industry. Artificial intelligence 
in the healthcare industry is expected to bring down the healthcare costs by 5 percent to 9 percent by using machine learning to tailor treatments and keep patients engaged, i.e. approximately $\$ 2$ trillion to $\$ 10$ trillion savings globally by tailoring drugs and treatments and 0.2 to 1.3 additional years of average life expectancy. ${ }^{4}$ The development of more advanced and affordable cloud computing infrastructures has a huge impact on the growth potential of Artificial intelligence. Drug discovery held over 35 percent of the global artificial intelligence market share and is anticipated to witness more than 40 percent growth rate over the period 2017 to 2027 . More than 86 percent of all healthcare spending is aimed at treating chronic diseases; Artificial intelligence technology could considerably change doctor-patient relationships. ${ }^{5}$

Artificial intelligence in healthcare has the potential to improve health outcomes by 30 percent to 40 percent whereas reducing healthcare costs by as much as 50 percent. ${ }^{6}$ The top three AI health applications that correspond to the maximum near-term value are robotassisted surgery, virtual nursing assistants and administrative workflow assistance with market valued of $\$ 40$ billion, $\$ 20$ billion and $\$ 18$ billion respectively. The top three AI health applications that correspond to the maximum near-term value are robot-assisted surgery, virtual nursing assistants and administrative workflow assistance with market valued of $\$ 40$ billion, $\$ 20$ billion and $\$ 18$ billion respectively. Cognitive robotics can integrate information from pre-op medical records with real-time operating metrics to physically guide and enhance the physician's instrument precision. AI can save 20 percent of nurse's time by avoiding unnecessary visits, which help the healthcare workers to focus more on care reach, institutional readiness, security, and workforce. ${ }^{7}$

The US is leading in AI in healthcare market followed by Europe. The US health Artificial intelligence market was $\$ 625$ million in 2016 and is expected to reach $\$ 16.9$ billion by end of 2027 , with a growth rate of 36 percent over the next ten years. Artificial intelligence can save $\$ 300$ billion in the US market using machine learning tools for population health forecasting.

\section{FUTURE CHALLENGES}

Artificial intelligence is expected to disrupt market, with its broad scope of applications and potential to redefine the working model of healthcare, automotive, finance, education, social welfare and research industry. In healthcare, alone AI market is predicated to surpass \$6.6 billion by $2025 .^{7}$ This expeditious advance in AI will outperform humans on more tasks and increases productivity. However, macroeconomically the AI-driven automation will change the structure of the industry and will cause many people to lose jobs and will make their skills redundant. A report on automation and economy predicts $47 \%$ of occupations will be replaced and $9 \%$ will become completely displaced. ${ }^{9}$ It is imperative that the government trains and educates the American workers for the jobs of the future in order to prevent the inequity caused by the technological innovations. The researches find that the jobs that are likely to be most affected are jobs with low wage (under \$20), low skill and lesseducation (under high school) It is possible that the benefits of the technological innovations may be limited to the privileged few in the society..$^{9}$ The impact of big data and AI has been called the $4^{\text {th }}$ industrial revolution. Technological advancements and machine-learning has progressed from enhancing sensory images to objectively diagnose an image. Dr. Jha predicts that with the AIs ability to analyze 1000's of images and diagnose with precision without burnout makes it a super specialist. This ability will conveniently replace pathologists and radiologists in the upcoming decade. ${ }^{10}$

AI analytically potential has made "prediction" costeffective. Prediction is beneficial in uncertainty; it allows us to make an informed decision. Any business thrives with tools that enables them to make a logical forecast and increases productivity. All human activities can be divided into these 5-components data, prediction, judgement, action and outcomes. A change in one variable cause other's to respond to the changed variable. ${ }^{11}$ In Healthcare AI is a substitute for a "prediction machine" with more prediction (early screening, detection) there is a potential for us to over diagnose patients. "Overdiagnosis" means we are giving patients unnecessary treatments and anxiety. Researchers designed an AI-algorithm and placed in a coronary care unit to detect a heart dysrhythmia. The machine detected $7 \%$ new cases of atrial fibrillation. These patients were treated and managed accordingly, however when health outcomes were compared the intervention vs nonintervention group there was no statistical significance. The author's concluded that although AI provides more sensitivity in detection it doesn't always improve outcome. This is another example of "overuse" of healthcare resources that increases health care costs and adds to resource wastage. ${ }^{12}$ It is important to do a cost-benefit analysis when making an investment in AI-technologies to curb the challenge of overuse.

As technology becomes more prevalent and we become more dependent upon them. It raises some serious ethical and legal issues regarding the safety and reliability of these hardware and software devices. (self-driving cars and IBM-Watson) At the same time without a standardized metric system we are unable to compare the performance and outcomes of these technologies. This poses a concern as it takes away human autonomy and makes us rely on these machines for making health decisions. After all a technology is only as good as the information that is fed into it. These tools "come with no guarantees of fairness, equitability, or even veracity". ${ }^{13}$ Without regulations and standards, it is hard for these AImachines to be widely implemented. It is also important to gain the public's trust for AI-system and remove the fear of marginalizing the low-income communities. Recently, the health and human services has passed a trusted exchange framework and common agreement to improve the health information exchange and interoperability between various health care institutions. 
It has been drafted to ease the flow of public health institutes wherever it is need and provide a continuum of care to the patient.

The Bloomberg report on the impact of AI on the market suggests that although AI-technologies will be the most disruptive class of technologies, this change will not be immediate. The Gartner Hype cycle for technologies suggests that at this point in time AI has reached its pinnacle of effectiveness (trough of disillusionment) and for the next 10 years AI will be presumed to solve all problems, but it will not meet these expectations. The report predicts that in the next 10 years AI will go through series of "trials and errors." This will lead to standardization of a model for a defined function. In the next 10 years, there will a proper infrastructure of software, hardware, deep neural networks that will provide us with the foundation to build upon. This is called the resetting "plateau" after which we will be able to fully utilize the benefits of AI. ${ }^{14}$

\section{Recommendations}

The current market predictions of AI suggest that globally AI healthcare adoption will have a CAGR of $42 \%$ and by 2025 , $90 \%$ of US hospitals will implement AI. ${ }^{15}$ The medical error causes 221,000 to 400,000 deaths annually. ${ }^{15}$ The AI- technologies provide us with a more efficient, precise and accurate infrastructure to ensure checks. This in turn will ensure better quality of care and patient safety. In the current US healthcare market, there are many uncertainties that will determine the course of the future investments in AI. There are three ways in which we can leverage AI-technology to enhance performance it is by investing in blockchains-data analytics (population health), revenue cycle and administrative automation. However, in the current US healthcare market there are many uncertainties (affordable care act, value-based reimbursement, low hospital volume). We advocate that while investing in AItechnology analyze the process flows carefully and understand whether you are trying to introduce specialized feature (IBM-Watson) or decreasing inefficiencies. Secondly, opt for local vendors who can provide you with better support and more useful features. Design the infrastructure on best practice models. Lastly, under the current challenges faced by hospitals AI recommend executives to tread towards cost-containment and weigh in on the cost-benefit analysis of the AItechnology when making an investment

Funding: No funding sources

Conflict of interest: None declared

Ethical approval: Not required

\section{REFERENCES}

1. Shen J, Zhang CJP, Jiang B, Chen J, Song J, Liu Z, et al. Artificial Intelligence Versus Clinicians in
Disease Diagnosis: Systematic Review. JMIR Med Inform. 2019;7(3):e10010.

2. Komaroff AL. Algorithms and the "art" of medicine. Am J Public Health 1982;72(1):10-12.

3. Kirkpatrick K, Kaul A, Artificial Intelligence for Healthcare Applications, Market Analysis and Forecast, Tractica, Boulder, CO, USA, 2017.

4. Bertalan M. Artificial Intelligence Will Redesign Healthcare. Medicalfuturist.com. The Medical Futurist, n.d. Web. Available at: https://medical futurist.com/artificial-intelligence-will-redesignhealthcare/. Accessed on 12 September 2016.

5. Bennett $\mathrm{C}$, Hauser $\mathrm{K}$. Artificial intelligence framework for simulating clinical decision-making: A Markov decision process approach. Artificial Intelligence Med. 2013;57(1):9-19.

6. Ahuja AS, The impact of artificial intelligence in medicine on the future role of the physician, Peer J, 2019;7:e7702.

7. Patel VL, Shortliffe EH, Stefanelli M, Szolovits P, Berthold MR, Bellazzi R, et al. The coming of age of artificial intelligence in medicine. Artificial Intelligence Med. 2017;46(1):5-17.

8. Artificial Intelligence in Healthcare Market | Global (AI) Analysis 2018-2026. (n.d.). Available at: https://www.inkwoodresearch.com/reports/artificialintelligence-in-healthcare-market. Accessed on 12 September 2019.

9. Preparing for the Future of Artificial Intelligence. 2016. Available at: https://www.whitehouse.gov/ sites/default/files/whitehouse_files/microsites/ostp/ NSTC/p reparing_for_the_future_of_ai.pdf. Accessed on 12 September 2019.

10. Jha S, Topol EJ. Adapting to artificial intelligence: radiologists and pathologists as information specialists. JAMA. 2016;316:2353-4.

11. Agrawal A, Gans J, Goldfarb A. Prediction machines: the simple economics of artificial intelligence. Boston, MA: Harvard Business Review Press. 2018.

12. Komorowski M, Celi LA. Will Artificial Intelligence Contribute to Overuse in Healthcare?*. Crit Care Med. 2017;45:912-3.

13. Beam AL, Kohane IS. Big data and machine learning in health care. JAMA. 2018;319;1317-8.

14. Kahn J. Alphabet's DeepMind Is Trying to Transform Health Care - But Should an AI Company Have Your Health Records? Available at: https://www.bloomberg.com/news/articles/2017-1128/alphabet-s-deepmind-is-trying-to-transformhealth-care-but-should-an-ai-company-have-yourhealth-records. Accessed on 12 November 2019.

15. Das, R. Five Technologies That Will Disrupt Healthcare By 2020. Available at: https://www. forbes.com/sites/reenitadas/2016/03/30/top-5technologies-disrupting-healthcare-by2020/\#13d3acf86826 Accessed on 26 April 2016.

Cite this article as: Patel D, Kore SA. Artificial intelligence: future impacts, challenges and recommendations on healthcare services. Int J Community Med Public Health 2020;7:1596-8. 